\title{
Caracterización fisiológica de tres razas de maíz peruano cultivadas con altos contenidos de boro
}

\section{Physiological characterization of three Peruvian maize landraces grown under high boron contents}

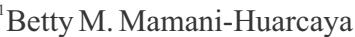 \\ ${ }^{2}$ Bret Luque-Zuñiga \\ ${ }^{3}$ Carlos J. Ceacero \\ 3," Jesús Rexach \\ Óscar Fernández Cutire
}

\author{
ORCID: 0000-0001-7389-9617 \\ ORCID: 0000-0002-0205-813X \\ ORCID: 0000-0001-9056-0906 \\ ORCID: 0000-0002-0392-8061 \\ ORCID: 0000-0002-2549-3046
}

\begin{abstract}
RESUMEN
El boro (B) es un nutriente esencial para un adecuado desarrollo de las plantas. En regiones áridas o semiáridas es frecuente encontrar suelos con elevado contenido de B. Este exceso de B desencadena problemas importantes de toxicidad en las plantas, que conducen a una reducción de su crecimiento y a una disminución de la fotosíntesis, entre otros efectos. El maíz es uno de los cultivos de mayor importancia en Perú. Muchas de las tierras de cultivo del sur del Perú presentan contenidos elevados de B. La selección de razas que sean tolerantes a estas condiciones es fundamental para obtener mejores rendimientos en las cosechas. El objetivo de este trabajo fue caracterizar la tolerancia al exceso de B de las razas de maíz Pachía, Lipe-Coruca y Sama, originarias de la región de Tacna (Perú). Para ello, se cultivaron estas razas de maíz en macetas con tierras con alto contenido de B (214 ppm), y fueron regadas con aguas del río Sama cuya concentración de B fue de $14.63 \mathrm{mgL}^{-1}$. Las razas Sama y Lipe-Coruca mostraron las mayores alturas con respecto al vástago. Además, la raza Sama presentó los más bajos contenidos foliares de B soluble e insoluble, y los mayores contenidos de clorofilas y carotenoides. En resumen, estos resultados sugieren que Sama es la raza más tolerante al exceso de B. Así, los menores contenidos de B y el mayor contenido de estos pigmentos de Sama permitirían una mejor adaptación al estrés generado por la acumulación de B en las tierras de cultivo.
\end{abstract}

Palabras clave: Lipe-Coruca, Pachía, Sama, Toxicidad de boro, Zea mays

\begin{abstract}
Boron (B) is an essential nutrient for proper plant development. It is common to find lands with high B contents in arid or semi-arid regions. Excess of B causes toxicity problems leading to stunted growth and a decline in photosynthesis rate, among other effects. Maize is one of the most important crops in Peru. Many of the cultivated land in southern Peru have high B contents. The selection of the races in these conditions is fundamental to improve maize crops. Therefore, the aim of this work was to determine the tolerance of B excess in Pachia, Lipe-Coruca, and Sama races of maize originating in Tacna, Peru. In consequence, the races of maize were cultivated in pots filled with high B contents (214 ppm), and watered with river water of Sama, which B concentration was $14.63 \mathrm{mg}$ L-1. Sama and Lipe-Coruca maizes showed the maximum stem heights. In addition, Sama maize presented the lowest foliar content of soluble and insoluble B, and the highest content of chlorophyll and carotenoid. In summary, the results suggest that the most tolerant land of B excess is Sama. Hence, the lowest contents of B and the highest pigment contents of Sama would allow a better adaptation of stress caused by the accumulation of B in cultivate lands.
\end{abstract}

Keywords: Lipe-Coruca, Pachía, Sama, Boron toxicity, Zea mays

\footnotetext{
Instituto Basadre de Investigación en Agrobiotecnología y Recursos Genéticos, Facultad Ciencias Agropecuarias, Universidad Nacional Jorge Basadre Grohmann, Tacna, Perú.

${ }^{2}$ Departamento de Agronomía, Facultad Ciencias Agropecuarias. Universidad Nacional Jorge Basadre Grohmann, Av. Cusco s/n, Tacna, Perú.

${ }^{3}$ Departamento de Fisiología, Anatomía y Biología Celular. Universidad Pablo de Olavide, E-41013, Sevilla, España.

${ }^{4}$ Departamento de Agronomía, Facultad Ciencias Agropecuarias, Universidad Nacional Jorge Basadre Grohmann, Tacna, Perú.
} 


\section{INTRODUCCIÓN}

El boro (B) es un micronutriente esencial para el correcto desarrollo de las plantas. Puede formar complejos con compuestos que contengan dos grupos hidroxilos en configuración cis (Landi et al., 2019). La función primordial que realiza el B en las plantas es estructural. Forma enlaces éster con los residuos de apiosa de dos moléculas de ramnogalacturonano II (RGII) en las paredes celulares (Kobayashi et al., 1996). Estos complejos de borato y RGII dan estabilidad a las paredes y son esenciales para el proceso de elongación celular (De Cnodder et al., 2005). De hecho, la falta de $\mathrm{B}$ provoca una rápida disminución de la elongación de la raíz de Arabidopsis thaliana (Princi et al., 2016).

$\mathrm{Al}$ ser el $\mathrm{B}$ un micronutriente, su rango óptimo de concentraciones en el suelo es muy pequeño (Landi et al., 2019). Es frecuente encontrar suelos donde las concentraciones de B se hallen en condiciones de deficiencia o toxicidad para las plantas. Además, las condiciones climáticas determinan el contenido de este nutriente en los suelos. En regiones áridas y semiáridas, la elevada evaporación del agua del suelo y la escasez de precipitaciones favorecen a la acumulación de $\mathrm{B}$ en las capas superiores del suelo. Así, en las regiones áridas, la toxicidad de $\mathrm{B}$ es uno de los problemas más importantes que pueden afectar al rendimiento de las cosechas (Princi et al., 2016; Landi et al., 2019).

Al igual que otros elementos, el B es tóxico para el desarrollo de las plantas cuando se acumula en el interior de las células vegetales a elevadas concentraciones. La alta permeabilidad del ácido bórico puede generar problemas de toxicidad cuando los suelos contienen altos niveles de B. Se considera que concentraciones de B en los suelos superiores $12 \mathrm{mg} \mathrm{kg}^{-1}$ causan efectos tóxicos en las plantas (Tanaka y Fujiwara, 2008). Así, cuando las plantas se cultivan en presencia de altas concentraciones de $\mathrm{B}$, se producen importantes alteraciones en $\mathrm{su}$ crecimiento vegetativo $\mathrm{y}$ reproductivo (Herrera-Rodríguez et al., 2010). De hecho, el exceso de $\mathrm{B}$ provoca una inhibición del crecimiento de los tallos y las raíces al disminuir su división y expansión celular, lo cual conducen a un menor desarrollo y tamaño de plantas (Nable et al., 1997). Además, el exceso de B provoca clorosis y/o manchas necróticas en las hojas. Estas necrosis se originan por la acumulación de B en las hojas de algunas especies debido al transporte de este nutriente a través de la corriente de transpiración del xilema (Camacho-Cristóbal et al., 2008). Los altos contenidos de B también afectan a la fotosíntesis, provocando una disminución del área fotosintética y de clorofila. Esto conlleva a una reducción de la tasa fotosintética (Landi et al., 2019; Simón-Grao et al., 2019). Otros efectos provocados por las altas concentraciones de B son alteraciones en el metabolismo del nitrógeno y en los niveles de sacarosa y almidón (Princi et al., 2016; Landi et al., 2019).

Pese a estos efectos tan drásticos generados por el exceso de B, las bases fisiológicas de su toxicidad no están claras. Sin embargo, el origen de su toxicidad podría deberse a su capacidad para unirse con moléculas clave que contengan grupos hidroxilos en configuración cis, especialmente moléculas de ribosa. De tal forma que el excedente de este nutriente se enlazaría con compuestos que poseen estos grupos, lo que provocaría una alteración en sus estructuras y/o funciones (Reid et al., 2004). Así, se han propuesto tres hipótesis para explicar la toxicidad del B en las plantas:

1) Afectando la estructura de la pared celular (De Cnodder et al., 2005).

2) Alterando el metabolismo energético mediante su unión a los residuos de ribosa de moléculas como el ATP, NADH y NADPH (Reid et al., 2004).

3) Finalmente, el exceso de B desorganizaría la división celular y el desarrollo por su unión a las ribosas libres o que forman parte del RNA (Reid et al., 2004).

De hecho, se ha sugerido que el B interferiría con la transcripción y/o traducción mediante su unión a ribosas que están expuestas durante el splicing del RNA y/o en el t-RNA(Reid, 2010).

La tolerancia de las plantas a la toxicidad de B varía entre especies e incluso entre variedades de la misma especie (Nable et al., 1997). Algunas plantas han desarrollado mecanismos que las hacen tolerantes a los altos contenidos de B. De hecho, esta resistencia está asociada a una menor absorción y a una más baja acumulación de este elemento en los tejidos de la planta (Princi et al., 2016; Landi et al., 2019). Así, se han propuesto dos estrategias que aumentan la tolerancia de las plantas al exceso de B: 
1) Se ha descrito un mecanismo activo de salida de $\mathrm{B}$ desde la raíz hacia el exterior, mediada por el transportador de B BOR4 (Wakuta et al., 2016). En A. thaliana, este transportador (AtBOR4) se localiza en el plasmalema de las células radicales y permite una excreción del exceso del B desde la raíz hacia el suelo.

2) Otra estrategia desarrollada es una represión post-transcripcional de los transportadores de B, AtNIP5;1 y AtBOR1 de Arabidopsis, en condiciones de alto B. Estas dos proteínas están implicadas en el transporte de B desde el suelo hasta el xilema en condiciones de deficiencia de B. La represión de estos dos transportadores reduce la cantidad de B que entra al xilema y, por tanto, al resto de los tejidos de la planta (Yoshinari y Takano, 2017).

El sur del Perú tiene una importante actividad agrícola, pero la productividad de sus cultivos se ve muy afectada por la escasez de lluvia. La precipitación media anual en esta región es de $28.1 \mathrm{~mm}$ (Pouyaud et al., 2001; PDRC, 2016). Así, para conseguir cosechas rentables es necesario introducir el regadío. En Tacna, existen las cuencas hidrográficas de los ríos Caplina, Sama, Locumba, Uchusuma y Caño, cuyas aguas se utilizan para el riego de los cultivos (ANA, 2015). Las aguas de las cuencas del Locumba y Sama son de baja calidad debido a la elevada actividad volcánica e hidrotermal y a los residuos de la minería. Varios ríos de las cuencas de los ríos Locumba y Sama presentan unos contenidos elevados de B (Franco et al., 2012). Por ejemplo, en puntos del cauce del río Salado (cuenca Locumba) se han medido concentraciones de B de $17.63 \mathrm{mg} \mathrm{L}^{-1}$. Además, en el río Sama se han medido concentraciones de B de 14.49 y $17.39 \mathrm{mg}$ $\mathrm{L}^{-1}$ (ANA, 2017). Las concentraciones máximas aceptables de B en las aguas para su consumo humano varían entre $5.0 \mathrm{mg} \mathrm{L}^{-1}$ (Canadá) hasta 0.3 mg L ${ }^{-1}$ (EE. UU.) (Kot, 2009). En Perú, los estándares Nacionales de la Calidad Ambiental del Agua (ECA) establecen un contenido de B de 1 $\mathrm{mg} \mathrm{L}^{-1}$ para el agua de riego de los cultivos vegetales, muy por debajo del contenido de B medido en estas cuencas (Decreto Supremo de la República del Perú, Nº 004-2017-MINAM).

Por tanto, teniendo en cuenta la importancia del maíz como alimento prioritario para la población humana y el ganado, el elevado contenido de B de estas tierras del sur del Perú y el efecto que este estrés provoca en el rendimiento de los cultivos; el objetivo de este trabajo fue analizar cómo afecta el exceso de B a tres razas de maíz. Se han medido diversos parámetros morfo-fisiológicos para determinar la raza más tolerante al exceso de $\mathrm{B}$.

\section{MATERIAL YMÉTODOS}

\section{Material vegetal}

Se utilizaron las razas de Zea mays Lipe-Coruca, Pachía y Sama. Estas tres razas fueron seleccionadas por los agricultores de la región de Tacna durante varias generaciones. En este proceso se obtuvieron las razas mejor adaptadas a las condiciones de estos suelos y a la baja calidad del agua de riego.

\section{Condiciones de cultivo}

Las semillas fueron remojadas durante 24 horas en agua destilada. Se sembraron 30 semillas por cada raza en bolsas de polietileno. Estas bolsas fueron rellenadas con $1 \mathrm{~kg}$ de suelo agrícola procedente del fundo del agricultor Lucio Martínez del distrito de Sama. Este suelo presentó un contenido de B de 214 ppm. Las plantas se regaron dos veces por semana, manteniendo la capacidad de campo, con agua procedente del río Sama con una concentración de B de $14.63 \mathrm{mg} \mathrm{L}^{-1}$. Las plántulas se mantuvieron durante 20 y 30 días en el invernadero del laboratorio de Biotecnología Vegetal de la Facultad de Ciencias Agropecuarias de la UNJBG de Tacna. Transcurrido este periodo, se recogieron seis plantas de cada raza de maíz y se separaron los vástagos de las raíces. Estos órganos se usaron para la determinación del contenido de $\mathrm{B}$ y de los pigmentos. El material vegetal se congeló hasta su posterior uso.

\section{Caracterización de parámetros morfológicos}

A los 20 y 30 días, tras la siembra de las semillas, se determinó el tamaño de los tallos y de los vástagos con una cinta métrica.

\section{Determinación de boro}

El contenido de B soluble e insoluble se determinó utilizando el método descrito por Fitzpatrick y Reid (2009) con algunas modificaciones. El material vegetal fue triturado hasta un fino polvo con un mortero, previamente enfriado en $\mathrm{N}_{2}$ líquido. E1 B contenido en unos 150-200 mg de material se extrajo con $500 \mu \mathrm{L}$ de agua de alta pureza a $80{ }^{\circ} \mathrm{C}$ durante $30 \mathrm{~min}$. Cada 10 minutos, las muestras se agitaron en un vórtex. 
Transcurrido este periodo, los viales se centrifugaron a $12500 \mathrm{~g}$ durante 10 minutos y los sobrenadantes se recolectaron; los precipitados se resuspendieron en otros $500 \mu \mathrm{L}$ de agua y se repitió el proceso. Los extractos acuosos (B soluble) y los precipitados (B insoluble) se congelaron hasta la determinación del B.

Los extractos acuosos se transfirieron a crisoles de porcelana, se secaron a $80^{\circ} \mathrm{C}$ durante 72 horas y se quemaron hasta cenizas en una mufla a $550{ }^{\circ} \mathrm{C}$ durante seis horas. Las cenizas se disolvieron en 1 $\mathrm{ml}$ de ácido clorhídrico $0.1 \mathrm{~N}$ y el contenido de boro fue determinado usando el método de $\mathrm{H}$ azometina.

Los precipitados (B insoluble) se pasaron a crisoles y se calentaron a $550^{\circ} \mathrm{C}$ durante seis horas en una mufla. Las cenizas se trataron como se ha descrito anteriormente para la determinación del B insoluble.

\section{Determinación de pigmentos}

La extracción de los pigmentos, a partir de material vegetal triturado, se realizó con acetona al $80 \%(\mathrm{v} / \mathrm{v})$. Se midió la absorbancia de los extractos acetónicos a 470, 647 y $663 \mathrm{~nm}$ para determinar la concentración de la clorofila $a$, clorofila $b$ y carotenoides (Lichtenthaler, 1987).

\section{Análisis estadísticos}

Los estadísticos fueron la media \pm la desviación estándar de seis plantas individuales. Los resultados fueron estadísticamente comparados usando el análisis unidireccional de varianza (ANOVA). Las diferencias significativas entre las razas $(P<0.05)$ se evaluaron mediante el uso de la prueba Tukey de diferencia honestamente significativa (post-hoc Tukey HSD).

\section{RESULTADOS}

\section{Determinación del tamaño del tallo y del vástago}

Los tallos de estas tres razas alcanzaron una longitud promedio de $12 \mathrm{~cm}$, no encontrándose diferencias en el crecimiento de este órgano en las tres razas analizadas (Fig. 1A). Sin embargo, cuando se determinó el tamaño del vástago se observaron diferencias significativas entre estas razas. Lipe-Coruca y Sama presentaron un mayor crecimiento de sus vástagos que Pachía (Fig. 1B).
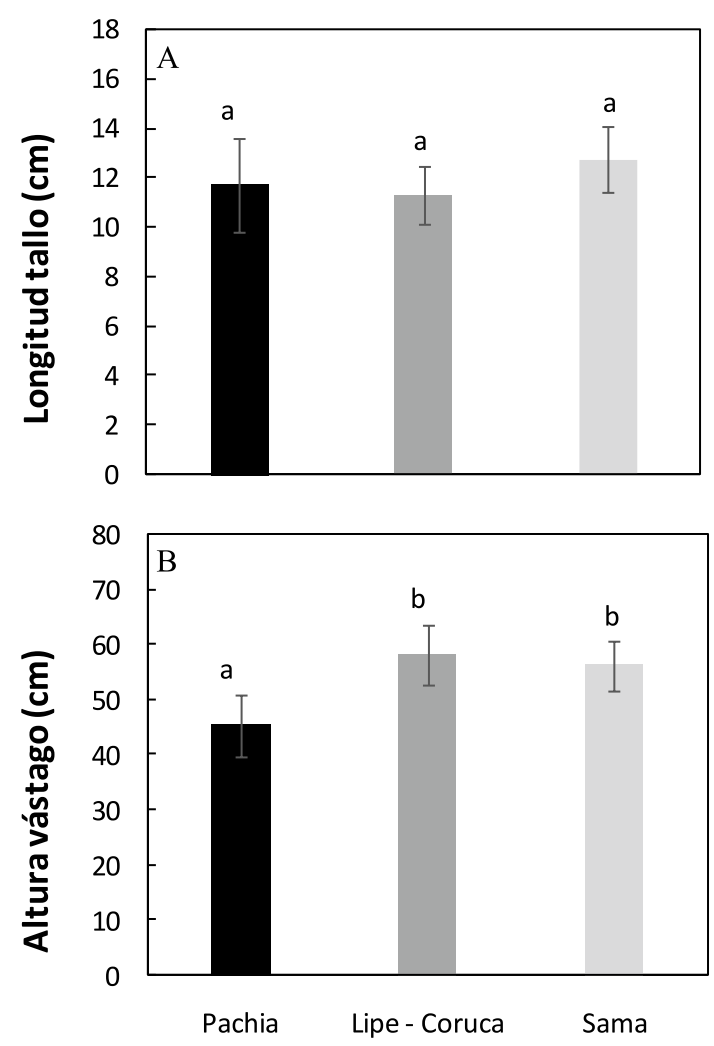

Figura 1. Longitud del tallo (A) y altura del vástago (B) de las razas de maíz Pachía, Lipe-Coruca y Sama a los 30 días desde su germinación. Nota: Los resultados son la media \pm desviación estándar de seis plantas. Diferentes letras indican las diferencias estadísticamente significativas $(P<0.05)$ entre las razas. El análisis estadístico se realizó con la prueba ANOVA con post-hoc test de Tukey HSD.

\section{Determinación del contenido de boro soluble e insoluble}

No se observaron diferencias significativas en la concentración radical de B soluble entre las tres razas analizadas (Fig. 2A). El contenido radical de B insoluble fue menor en Sama (Fig. 2B). Interesantemente, en las hojas, la concentración de B soluble fue significativamente menor en las razas Lipe-Coruca, pero sobre todo en Sama, donde los niveles medidos fueron de $0.67 \mathrm{mg} / \mathrm{g}$ (PF) (Fig. 3A). Se observó el mismo patrón en el B insoluble donde los valores más bajos se obtuvieron en Sama (Fig. 3B). Por tanto, Sama es capaz de acumular niveles más bajos de $\mathrm{B}$, especialmente de B soluble en sus hojas. 

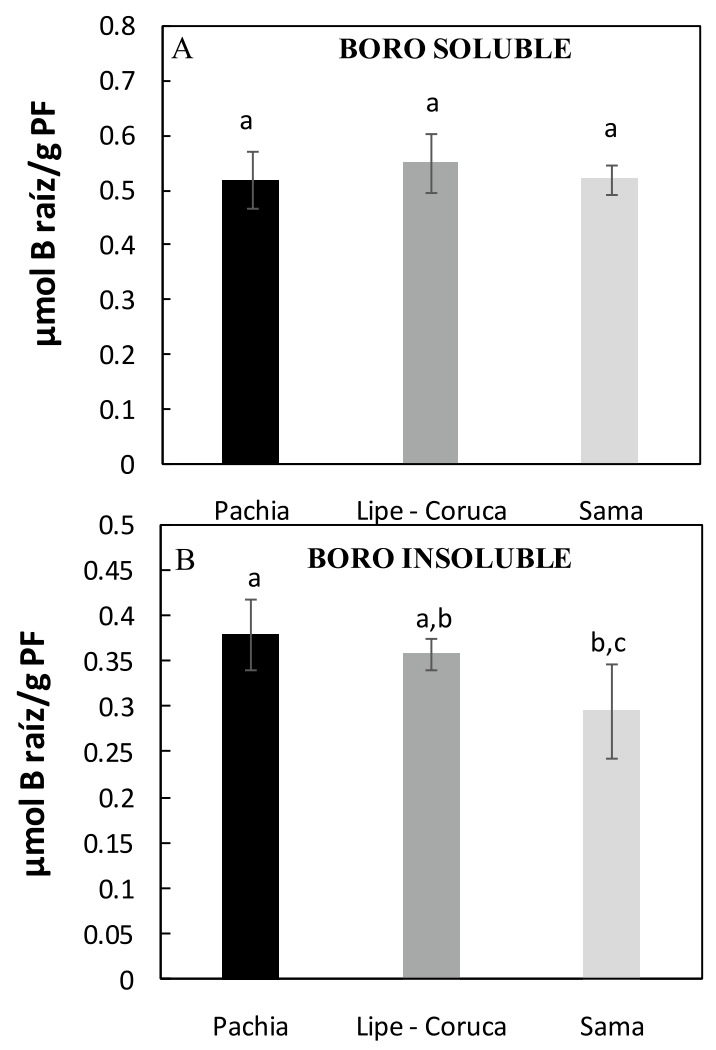

Figura 2. Concentración de boro soluble (A) e insoluble (B) en las raíces de las razas de maíz Pachía, Lipe-Coruca y Sama a los 20 días desde su germinación.

Nota: Para más detalles de la extracción y determinación de boro ver Materiales y Métodos. Los resultados son la media \pm desviación estándar de seis plantas. Diferentes letras indican las diferencias estadísticamente significativas $(P<0.05)$ entre las razas. El análisis estadístico se realizó con la prueba ANOVAcon post-hoc test de Tukey HSD.

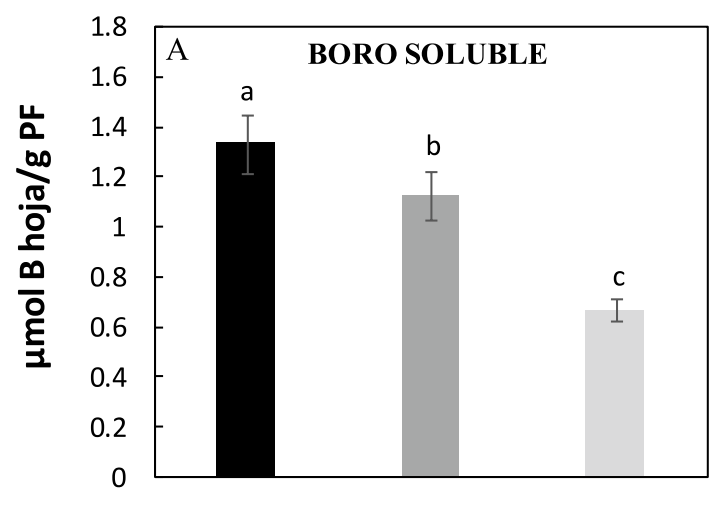

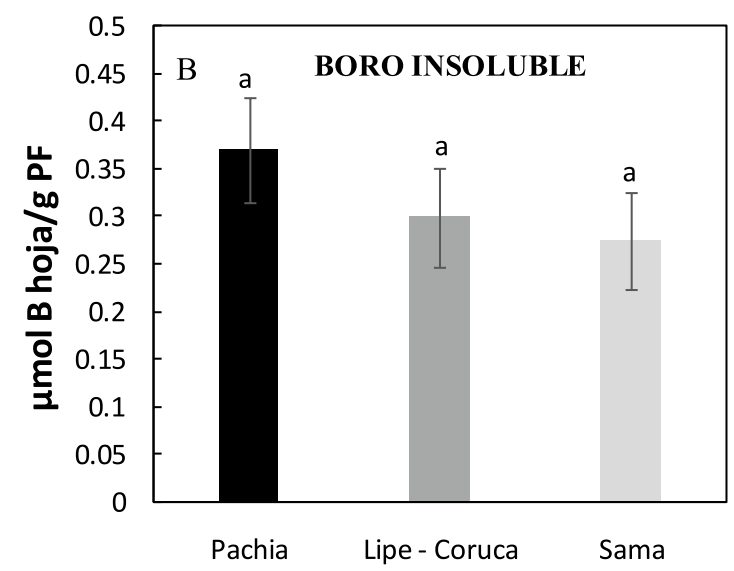

Figura 3. Concentración de boro soluble (A) e insoluble (B) en las hojas de las razas de maíz Pachía, Lipe-Coruca y Sama a los 20 días desde su germinación.

Nota: Para más detalles de la extracción y determinación de boro ver Materiales y Métodos. Los resultados son la media \pm desviación estándar de seis plantas. Diferentes letras indican las diferencias estadísticamente significativas $(P<0.05)$ entre las razas. El análisis estadístico se realizó con la prueba ANOVA con post-hoc test de Tukey HSD.

\section{Determinación del contenido de pigmentos}

Las concentraciones de clorofila $a$ y $b$ fueron significativamente mayores en Sama. Además, Pachía fue la raza que presentó los valores más bajos (Figuras 4A y 4B). Como era de esperar, el contenido de clorofila total (calculado como la suma de clorofila $a$ y $b$ ) fue mayor en Sama y los valores más pequeños se obtuvieron en Pachía (Figura 4C).

Los niveles de carotenoides fueron muy similares en Pachía y Lipe-Coruca y significativamente mayores en Sama, donde se alcanzó una concentración de $0.35 \mathrm{mg} / \mathrm{g}(\mathrm{PF})$.

En resumen, la variedad Sama presentó unos contenidos de clorofila $a$ y $b$ y de carotenoides marcadamente mayores que los obtenidos en las otras dos razas (Figuras 4 y 5 ).

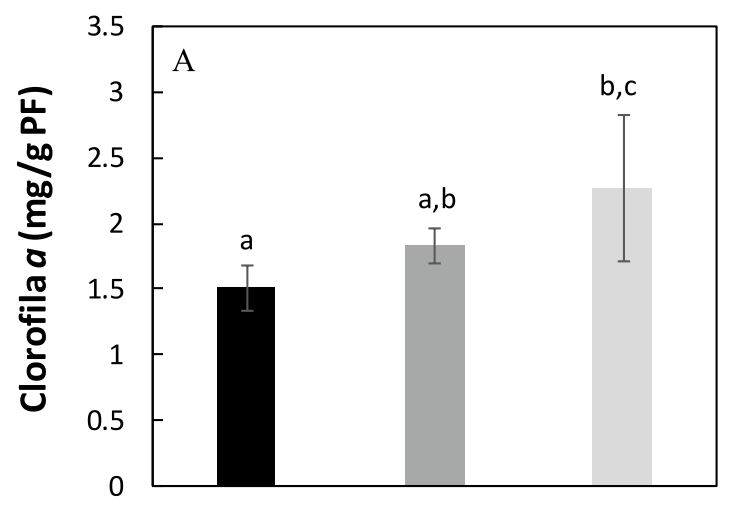



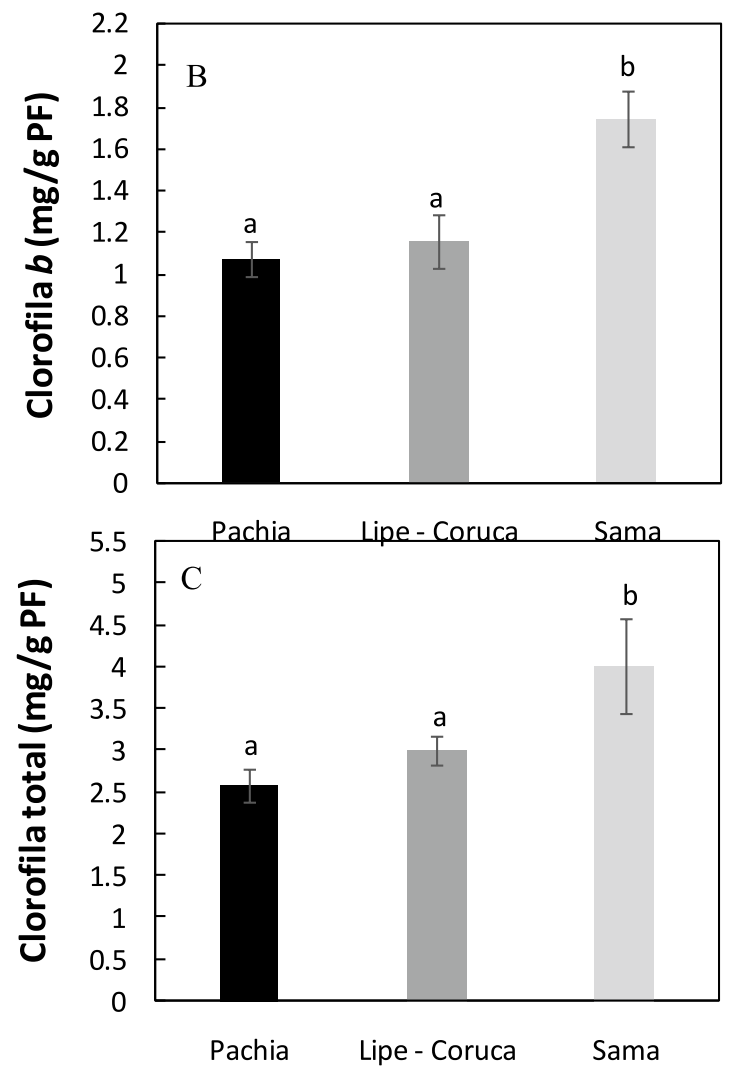

Figura 4. Concentración de clorofila $a$ (A), clorofila $b$ (B) y clorofila total (C) en las razas de maíz Pachía, Lipe-Coruca y Sama a los 20 días desde su germinación.

Nota: Diferentes letras indican las diferencias estadísticamente significativas $(P<0.05)$ entre las razas. El análisis estadístico se realizó con la prueba ANOVAcon post-hoc test de Tukey HSD.

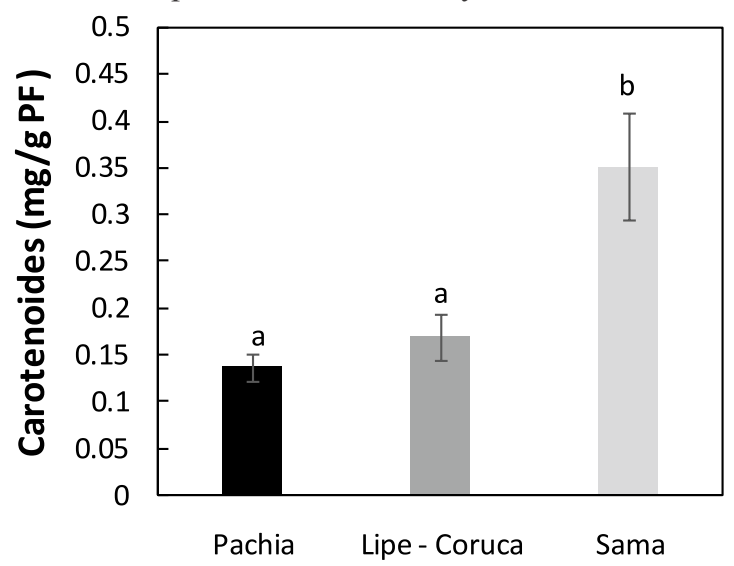

Figura 5. Concentración de carotenoides en las razas de maíz Pachía, Lipe-Coruca y Sama a los 20 días desde su germinación.

Nota: Para más detalles ver Materiales y Métodos. Los resultados son la media \pm desviación estándar de seis plantas. Diferentes letras indican las diferencias estadísticamente significativas $(P<0.05)$ entre las razas. El análisis estadístico se realizó con la prueba ANOVA con post-hoc test de Tukey HSD.

\section{DISCUSIÓN}

El elevado contenido de B en las tierras de cultivo es un problema importante a nivel mundial que condiciona el correcto desarrollo de los cultivos, así como su rendimiento (Nable et al., 1997; Princi et al., 2016; Landi et al., 2019). El sur del Perú es una región extremadamente seca con una importante superficie dedicada a la agricultura que deja un significativo impacto económico en la región (Pouyaud et al., 2001). Este clima seco junto con la intensa actividad volcánica genera una acumulación de $\mathrm{B}$ en los cultivares. En muchos casos, estas concentraciones de B exceden las cantidades que los cultivos pueden tolerar, lo que conduce a una merma de su desarrollo y de su rentabilidad económica. Perú posee una riqueza enorme de razas/variedades de Zea mays, que durante cientos de miles de años, han sido cultivadas y seleccionadas por los agricultores con la finalidad de obtener aquellas más productivas.

El objetivo de este trabajo ha sido realizar una primera caracterización fisiológica de tres razas de maíz para analizar cuál de estas puede ser más tolerante a los altos contenidos de B en los suelos. Así, se han cultivado las razas Pachía, LipeCoruca y Sama en suelos agrícolas procedentes de cultivares de la región de Sama, que se caracterizan por su alto contenido de B (214 ppm). Estas plantas fueron regadas con aguas del río Sama, caracterizada también por el elevado contenido de B (14.63 $\left.\mathrm{mg} \mathrm{L}^{-1}\right)$. Bajo estas condiciones de cultivo, las razas Sama y LipeCoruca tuvieron un mayor crecimiento de sus vástagos que Pachía (Fig. 1B). Entre los efectos que provoca el exceso de $\mathrm{B}$, en las plantas destacan el retraso en el desarrollo, la reducción del tamaño de los vástagos y del crecimiento radical (Nable et al., 1997; Herrera-Rodríguez et al., 2010). Se ha propuesto que la disminución de las divisiones de las células de los meristemos radicales y la inhibición de la expansión de la pared celular, inducidas por la toxicidad de B, serían la causa que conduciría al menor crecimiento de los vástagos y las raíces observado bajo estas condiciones (Herrera-Rodríguez et al., 2010). Por tanto, el mayor tamaño de los vástagos de Sama y Lipe-Coruca indicaría que estas razas son más tolerantes al alto contenido de $\mathrm{B}$ de estos suelos y agua de riego.

Se determinaron los contenidos de B soluble e insoluble en hojas y raíces. Las concentraciones de $\mathrm{B}$ han sido usadas como un indicador para 
determinar la tolerancia de las plantas frente al exceso de B. De hecho, una de las características de las plantas tolerantes al exceso de B es la menor acumulación de este nutriente en sus tejidos. Por ejemplo, en trigo y cebada la tolerancia al exceso de $\mathrm{B}$ se relaciona con una menor acumulación de $\mathrm{B}$ en los vástagos (Nable, 1988; Sutton et al., 2007). El reducido contenido de B en los tejidos de las plantas tolerantes puede ser debido a tres mecanismos no excluyentes:

1) Una disminución de la absorción de B en las raíces que conduce a un menor contenido de este nutriente en las raíces y vástagos.

2) Un mecanismo de transporte activo de excreción de B.

3) Una disminución de la velocidad de transpiración que conllevaría a un menor transporte del $\mathrm{B}$ desde las raíces hacia los vástagos (Camacho-Cristóbal et al., 2008; Princi et al., 2016; Macho-Rivero et al., 2017). Los resultados obtenidos en este trabajo mostraron que Sama presentó unos niveles foliares de B soluble significativamente menores que las otras dos razas. Sin embargo, en las raíces solo fueron significativamente menores los contenidos de B insoluble (Figuras 2 y 3 ). Estos datos sugieren que la cantidad de B absorbida por las raíces en estas tres razas debe de ser similares, dado que el contenido de B soluble radical es muy parecido en las tres razas. Además, bajo estas condiciones de cultivo, no podría ser excluida la existencia un mecanismo de excreción activa de $\mathrm{B}$ desde las raíces al suelo que sea específico o que esté más activo en una de estas razas. Interesantemente, en las hojas, los menores niveles de B soluble de Lipe-Coruca y, sobre todo, en Sama sugieren que estas razas tienen disminuido el transporte de B hacia los vástagos. Así, ambas razas podrían tener una reducida carga de $\mathrm{B}$ al xilema que explicaría los menores niveles de B observados. El transporte de B en las raíces y su posterior carga al xilema se realiza por difusión pasiva y mediante una absorción facilitada por varios transportadores/canales (Camacho-Cristóbal et a1., 2008; Tanaka y Fujiwara, 2008). En Arabidopsis thaliana, entre estos transportadores/canales se encuentran NIP5; 1 que se requiere en la absorción de $\mathrm{B}$ en las raíces y BOR1 implicado en la carga del B al xilema (Takano et al., 2002; Takano et al., 2006). Además, el transportador BOR4 de Arabidopsis excreta B desde la raíz hacia el suelo (Wakuta et al., 2016). En esta especie, se han propuesto dos estrategias que aumentarían su tolerancia al exceso de B. Por un lado, el mecanismo activo de eflujo de $\mathrm{B}$ desde la raíz hacia el exterior, mediada por el transportador de B AtBOR4 que permite una excreción del exceso del $\mathrm{B}$ desde la raíz hacia el suelo (Wakuta et al., 2016). Por otro lado, una represión post-transcripcional de AtNIP5;1 y AtBOR1 en condiciones de alto B. Estos dos transportadores de Arabidopsis están implicados en el transporte de B desde el suelo hasta el xilema, al reprimirlos, se reduce la cantidad de B que entra al xilema $y$, por tanto, al resto de los tejidos de la planta (Yoshinari et al., 2017). LipeCoruca y, principalmente, Sama podrían disminuir la cantidad de $\mathrm{B}$ transportada desde las raíces hasta los vástagos, disminuyendo la actividad de transportadores/canales ortólogos a AtNIP5; 1 y AtBOR1. En el genoma de Zea mays se han identificado varios genes que presentan homología con AtBOR1 y AtNIP5;1. Sería interesante analizar la expresión de estos genes, en estas tres razas, para ver si Lipe-Coruca y Sama presentan una expresión diferencial bajo estas condiciones de cultivo.

Como se ha comentado anteriormente, otro de los mecanismos mediante los cuales las plantas pueden reducir los contenidos de $\mathrm{B}$ en sus tejidos aéreos es reduciendo la transpiración. Por tanto, los menores contenidos foliares de B soluble en Lipe-Coruca y, sobre todo, en Sama también podrían deberse a que estas razas tendrían una velocidad de transpiración más reducida. Esto conduciría a una reducción del transporte de B hacia los vástagos y a un menor contenido de $\mathrm{B}$ (Fig. 3). De hecho, el transporte de B a las hojas es realizado primeramente a través del xilema y conducido hasta las partes aéreas de la plantas por la corriente de transpiración (Nable et al., 1997). $\mathrm{Se}$ ha mostrado una relación directa entre la velocidad de transpiración y la acumulación de B en las hojas (Macho-Rivero et al., 2018).

Cuando se analizaron los contenidos de pigmentos en estas tres razas se observó que Sama fue la única que presentó diferencias significativas en los contenidos de clorofila $a$, clorofila $b$ y clorofila total (Fig. 4). La fotosíntesis es otro de los parámetros que se ve afectado cuando las plantas se someten a altas concentraciones de B (Landi et al., 2012, 2019). Elevadas concentraciones de $\mathrm{B}$ reducen el contenido de clorofilas y la tasa de fotosíntesis en muchas especies (Sarafi et al., 2018; Kaya et al., 2019; Landi et al., 2019; Simón-Grao et al., 2019; Choudhary et al., 2020). Pese a que no se conoce en detalle los mecanismos implicados en la 
alteración de la fotosíntesis por este estrés, esta reducción en la asimilación de $\mathrm{CO}_{2}$ parece estar relacionada con una combinación de diferentes factores: acumulación de especies reactivas de oxígeno (ROS), disminución en las actividades de las enzimas fotosintéticas y velocidad del transporte de electrones (Landi et al., 2012). La generación de ROS provocada por el exceso de B en los medios puede explicar el menor contenido de clorofilas observado bajo estas condiciones (Landi et al., 2012). Así, los mayores contenidos de clorofilas en Sama podrían proteger mejor a esta raza de la degradación de las clorofilas observadas bajo este estrés y permitirían una mejor adaptación de esta raza. Por otro lado, es bien conocido el efecto antioxidante de los carotenoides que reducen los efectos nocivos de la acumulación de ROS. Así, el mayor contenido de carotenoides de Sama (Fig. 5) contribuiría a una mayor protección de esta raza frente al exceso de B.

\section{CONCLUSIONES}

Los resultados mostrados en este trabajo sugieren que la raza de maíz Sama es más tolerante a los elevados contenidos de B en el suelo y agua de riego. Esta tolerancia podría ser debido a su capacidad para reducir la cantidad de B transportado desde el suelo hasta el xilema y/o a su capacidad para disminuir la velocidad de transpiración bajo estas condiciones. De este modo, podría reducirse los contenidos de $\mathrm{B}$ en sus vástagos hasta niveles menos tóxicos. Además, el mayor contenido de clorofilas y carotenoides permitiría una mejor adaptación de esta raza al estrés generado por la acumulación de $\mathrm{B}$ en el suelo.

\section{AGRADECIMIENTOS}

Este trabajo ha sido financiado por el Proyecto 2016SEC014 de la Agencia Andaluza de Cooperación Internacional para el Desarrollo de la Junta de Andalucía (España). Además, se ha realizado gracias al convenio de colaboración aprobado por Resolución Rectoral No 2662-2017UN/JBG, entre la Universidad Pablo de OlavideSevilla (España) y la Universidad Nacional Jorge Basadre Grohmann-Tacna (Perú), a través del Instituto Basadre de Investigación en Agrobiotecnología y Recursos Genéticos (IRGAB).

\section{REFERENCIAS}

ANA (Autoridad Nacional del Agua). (2017). Informe de la Autoridad Nacional del Agua. Administración local del agua CaplinaLocumba. Remitido con oficio $\mathrm{N}^{\circ} 1227-$ 2017-ANA-AAA.CO-ALA.CL. Ministerio de Agricultura y Riego del Perú.

ANA (Autoridad Nacional del Agua). (2015). Plan de Gestión de los Recursos Hídricos de la Cuenca Caplina-Locumba, Autoridad Nacional del Agua, Lima: SOLVIMA GRAF S.A.C. ISBN: 978-612-4273-04-9. Disponible en www.ana.gob.pe.

Camacho-Cristóbal, J., Rexach, J. y González-Fontes, A. (2008). Boron in plants: deficiency and toxicity. J. Integr. Plant Biol., 50(10), 12471255 . doi: $10.1111 / \mathrm{j} .1744$ 7909.2008.00742.x.

Choudhary, S., Zehra, A., Naeem, M., Khan, M. y Aftab, T. (2020). Effects of boron toxicity on growth, oxidative damage, antioxidant enzymes and essential oil fingerprinting in Mentha arvensis and Cymbopogon flexuosus. Chem. Biol. Technol. Agric., 7, 8. doi: 10.1186/s40538-019-0175-y.

De Cnodder, T., Vissenberg, K., Van Der Straeten, D. y Verbelen J.-P. (2005). Regulation of cell length in the Arabidopsis thaliana root by the ethylene precursor1-aminocyclopropane-1carboxylic acid: a matter of apoplastic reactions. New Phytol., 168(3), 541-550. doi: 10.1111/j.1469-8137.2005.01540.x.

Fitzpatrick, K. y Reid, R. (2009). The involvement of aquaglyceroporins in transport of boron in barley roots. Plant Cell Environ, 32(10), 1357-1365. doi: 10.1111/j.13653040.2009.02003.x.

Franco, A., Ponce, S. y Rodríguez, J. (2012). Actividad hidrogeológica del sur del Perú, una evaluación situacional de las cuencas de Sama y Locumba. Tecnia, 22(1), 43-54. doi: 10.21754/tecnia.v22i1.89.

Herrera-Rodríguez, M., González-Fontes, A., Rexach, J., Camacho-Cristóbal, J., Maldonado, J. y Navarro-Gochicoa, M.T. (2010). Role of boron in vascular plants and response mechanisms to boron stresses. Plant Stress, 4(2), 115-122.

Kaya, C., Sarioğlu, A., Akram, N. y Ashraf, M. (2019). Thiourea-mediated nitric oxide production enhances tolerance to boron toxicity by reducing oxidative stress in bread wheat (Triticum aestivum L.) and durum wheat (Triticum durum Desf.) plants. J. Plant Growth Regul, 38, 1094-1109. doi: 10.1007/s0034 4-019-09916-x.

Kobayashi, M., Matoh, T. y Azuma, J. (1996). Two chains of rhamnogalacturonan II are crosslinked by borate-diolester bonds in higher 
plant cell walls. Plant Physiol, 110(3), 10171020. doi: 10.1104/pp.110.3.1017.

Kot, F. (2009). Boron sources, speciation and its potential impact on health. Rev. Environ. Sci. Bio-Technol, 8(1), 3-28, doi: 10.1007/s11157-008-9140-0.

Landi, M., Degl'Innocenti, E., Pardossi, A. y Guidi, L. (2012). Antioxidant and photosynthetic responses in plants under boron toxicity: a review. Am. J. Agr. Biol. Sci., 7(3), 255-270. doi: 10.3844/ajabssp.2012.255.270.

Landi, M., Margaritopoulou, T., Papadakis, I. y Araniti, F. (2019). Boron toxicity in higher plants: an update. Planta, 250: 1011-1032. doi: 10.1007/s00425-019-03220-4

Lichtenthaler, H.K. (1987). Chlorophylls and carotenoids: Pigments of photosynthetic biomembranes. Method. Enzymol., 148, 350382. doi: 10.1016/0076-6879(87)48036-1.

Macho-Rivero, M., Camacho-Cristóbal, J., HerreraRodríguez, M.B., Müller, M., Munné-Bosch, S. y González-Fontes, A. (2017). Abscisic acid and transpiration rate are involved in the response to boron toxicity in Arabidopsis plants. Physiol. Plant., 160(1), 21-32. doi: $10.1111 / \mathrm{ppl} .12534$.

Macho-Rivero, M. Herrera-Rodríguez, M.B., Brejcha, R., Schäffner, A., Tanaka, N., Fujiwara, T., González-Fontes, A. y Camacho-Cristóbal, J. (2018). Boron toxicity reduces water transport from root to shoot in Arabidopsis plants. Evidence for a reduced transpiration rate and expression of major PIP aquaporin genes. Plant Cell Phys iol., 59 ( 4 ), $841-849$. doi:10.1093/pcp/pcy026.

Nable, R. (1988). Resistance to boron toxicity amongst several barley and wheat cultivars: a preliminary examination of the resistance mechanism. Plant Soil, 112, 45-52, doi: 10.1007/BF02181751.

Nable, R., Banuelos, G. y Paull, J. (1997). Boron toxicity. Plant Soil, 193, 181-198. doi: 10.1023/A:100427222788.

PDRC. Plan de Desarrollo Regional ConcertadoTacna hacia el 2021. (2016). Aprobado con Ordenanza Regional No: 001-2016CR/GOB.REG.TACNA. Disponible en: http://www.regiontacna.gob.pe.

Pouyaud, B., Yerrén Suarez, J., Arboleda Orozco, J.F. y Suarez Alayza, W.A. (2001). Variabilidad pluviométrica, a escalas anual y cuatrimestral, en la vertiente peruana del océano Pacífico. Informe IRD-SENAMHI.

Princi, M., Lupini, A., Araniti, F., Longo, C., Mauceri, A., Sunseri, F. y Abenavoli, M.R.(2016). Boron toxicity and tolerance in plants: recent advances and future perspectives. En: Ahmad P, editor. Plant Metal Interaction: Emerging Remediation Techniques. Amsterdam: Elsevier Inc. ISBN: 978-0-12803158-2 doi: 10.1016/B978-0-12-8031582.00005-9.
Reid, R. (2010). Can we really increase yields by making crop plants tolerant to boron toxicity? Plant Sci., 178(1), 9-11. doi: 10.1016/j.plantsci.2009.10.006.

Reid, R., Hayes, J.E., Post, A., Stangoulis, J.C.R. y Graham, R. (2004). A critical analysis of the causes of boron toxicity in plants. Plant Cell Environ, 27(11):1405-1414. doi: 10.1111/j.1365-3040.2004.0124.

Sarafi, E., Siomos, A., Tsouvaltzis, P., Therios, I. y Chatzissavvidis, C. (2018). Boron toxicity effects on the concentration of pigments, carbohydrates and nutrient elements in six non-grafted pepper cultivars (Capsicum annuum L.). Ind. J. Plant Physiol. 23(3), 474-485. doi: 10.1007/s40502-018-0388-2.

Simón-Grao, S., Nieves, M., Cámara-Zapata, J., Martínez-Nicolás, J., Rivero, R., FernándezZapata, J. y García-Sánchez, F. (2019). The Forner Alcaide $\mathrm{n}^{\circ} 5$ citrus genotype shows a different physiological response to the excess of boron in the irrigation water in relation to its two genotype progenitors. $S c i$. Hortic., $245,19-28$, d o i : 10.1016/j.scienta.2018.10.002.

Sutton, T. Baumann, U., Hayes, J., Collins, N.C., Shi, B.J., Schnurbusch, T., Hay, A., Mayo, G., Pallotta, M., Tester, M. y Langridge, P.(2007). Boron-toxicity tolerance in barley arising from efflux transporter amplification. Science, 318(5855), 1446-1449. doi: $10.1126 /$ science. 1146853 .

Takano, J., Noguchi, K., Yasumori, M., Kobayashi, M., Gajdos, Z., Miwa, K., Hayashi, H., Yoneyama, T. y Fujiwara, T. (2002). Arabidopsis boron transporter for xylem loading. Nature, 420(6913), 337-340. doi: 10.1038 /nature01139.

Takano, J., Wada, M., Ludewig, U., Schaaf, G., Von Wirén, N. y Fujiwara, T. (2006). The Arabidopsis major intrinsic protein NIP5; 1 is essential for efficient boron uptake and plant development under boron limitation. Plant Cell, 18, $1498-1509$. d o i : 10.1105/tpc.106.041640.

Tanaka, M. y Fujiwara T. (2008). Physiological roles and transport mechanisms of boron: perspectives from plants. Eur. J. Phy., 456, 671-677. doi: 10.1007/s00424-007-0370-8.

Wakuta, S., Fujikawa, T., Naito, S. y Takano, J. (2016). Tolerance to excess-boron conditions acquired by stabilization of a BOR 1 variant with weak polarity in Arabidopsis. Front. Cell. Dev. Biol., 4, 4. doi: 10.3389/fcell.2016.00004.

Yoshinari, A. y Takano, J. (2017). Insights into the mechanisms underlying boron homeostasis in Plants. Front. Plant Sci., 8, 1951. doi: 10.3389/fpls.2017.01951. 